\title{
Pressure Distributions Generated along a Self-Acting Fluid-Lubricated Herringbone-Grooved Journal Bearing with Trapezoidal Groove
}

\author{
Jun Liu, ${ }^{1}$ and Yoshihiro Mochimaru ${ }^{2}$ \\ ${ }^{1}$ Tochigi Research \& Development Center, Keihin Corporation, 2021-8 Hoshakuji, Takanezawa-machi, Shioya-gun, \\ Tochigi 329-1233, Japan \\ ${ }^{2}$ Department of International Development Engineering, Graduate School of Science and Engineering, \\ Tokyo Institute of Technology, Meguro-ku, Tokyo 152-8550, Japan
}

Correspondence should be addressed to Jun Liu; liujun3@hotmail.com

Received 31 October 2012; Accepted 20 November 2012

Academic Editors: M. Dular and L. Qian

Copyright (C) 2013 J. Liu and Y. Mochimaru. This is an open access article distributed under the Creative Commons Attribution License, which permits unrestricted use, distribution, and reproduction in any medium, provided the original work is properly cited.

Numerical studies are carried out to investigate pressure distributions of a fluid-lubricated herringbone-grooved journal bearing with trapezoidal grooves of various angles. Additionally, the optimal trapezoidal groove geometry is discussed in terms of the radial load capacity and friction torque.

\section{Introduction}

Recently, herringbone-grooved journal bearings have a lot of applications on small rotating mechanisms such as hard disk and turbo machinery. As for a herringbone-grooved gas journal bearing under a narrow groove theory, Vohr and Pan [1] obtained numerical solutions for a special case of small eccentricity. Cheng and Pan [2] gave time-dependent solution of a nonlinear Reynolds equation under stable operation parameters for gas-lubricated bearings. Hamrock and Fleming [3] investigated optimal conditions of radial load capacity for self-acting herringbone-grooved journal bearings. The film in an incompressible fluid was analyzed numerically by Murata et al. [4] based on the potential flow theory. Bonneau and Absi [5] applied a finite element method (FEM) to a compressible Reynolds equation to get aerodynamic characteristics at 4 through 16 grooves with moderate eccentricity. Rondonuwu and Winoto [6] measured pressure distributions along hydrodynamic herringbonegrooved journal bearing for several groove patterns.

A fluid-lubricated herringbone-grooved journal bearing with trapezoidal grooves was introduced by Liu and Mochimaru [7], and the influence of viscous trapezoidal grooves on the bearing was evaluated later for various trapezoidal angles [8].

In this paper, the pressure distributions generated along a self-acting fluid-lubricated herringbone-grooved journal bearing with trapezoidal grooves were numerically investigated, using a spectral finite difference scheme. In addition, the optimal trapezoidal groove geometry is discussed in terms of the radial load capacity and friction torque.

\section{Analytical Model}

Consider a fluid-lubricated journal bearing equipped with herringbone groove as shown in Figure 1. The bearing length is $2 l$ and the groove is symmetric with respect to its center of bearing. The shaft itself rotates around its center $\mathrm{O}^{*}$ with an angular velocity $\omega$ in the counterclockwise direction and revolves around the center $O$ of the bearing with an angular velocity $\Omega$ in the counterclockwise direction. The eccentricity of the shaft is given by $\overline{O O^{*}}=e$, and the outer bearing is fixed.

The radius of bearing is $R_{b}$, the radius of shaft ignoring grooves is $R_{s 0}$, the bearing clearance $C_{r}$ is defined as $C_{r}=$ $R_{b}-R_{s 0}$, and the groove depth, the groove width, and ridge width, and the groove angle are denoted by $\delta, a_{g}, a_{r}$, and $\beta$, 


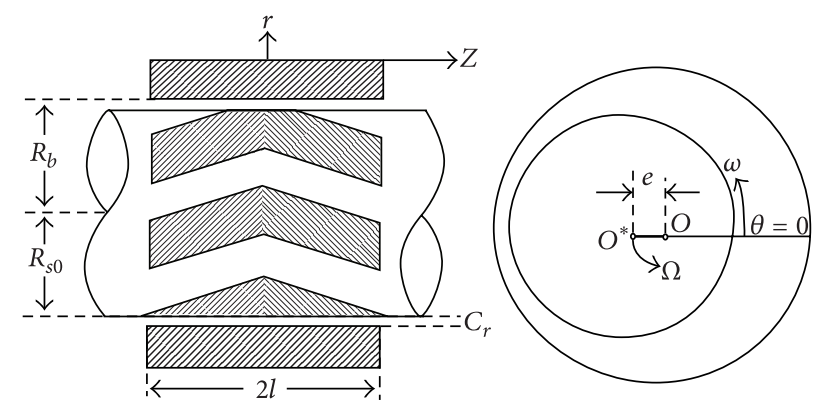

FIGURE 1: Herringbone-grooved journal bearing.

respectively. For a trapezoidal groove, the groove geometry is characterized by the angles $\psi_{l}$ and $\psi_{r}$ as shown in Figure 2, where $\widetilde{\psi}_{l}$ and $\widetilde{\psi}_{r}$ are the apparent trapezoidal angles of groove in $r \theta$ cross-section.

Two coordinate systems, $S$ and $S^{*}$, are introduced as follows.

(1) An inertial coordinate system $S(r, \theta, z)$ is fixed in the center $O$ of the outer bearing.

(2) A noninertial coordinate system $S^{*}\left(r^{*}, \theta^{*}, z^{*}\right)$ is fixed at the center $O^{*}$ of the rotation shaft.

Hereafter the superscript $*$ is meant for the non-inertial coordinate system.

\section{Pressure Distribution Equation and Spectral Finite Difference Method}

The pressure distribution in fluid film, $P$, can be obtained through a modified Reynolds equation with curvature effect derived by Liu and Mochimaru [7] as

$$
\begin{aligned}
\frac{\partial}{\partial \Theta}\left[(1+H) H^{3} \frac{\partial P}{\partial \Theta}\right]+\frac{\partial}{\partial Z}\left[H^{3} \frac{\partial P}{\partial Z}\right] \\
=-6 \sigma U_{s}\left(1-\frac{H}{2}\right)+3 \sigma V_{s}\left(1+H+\frac{5}{4} H^{2}\right) \frac{\partial H}{\partial \Theta} \\
+3 \sigma\left(H+\frac{H^{2}}{2}\right) \frac{\partial V_{s}}{\partial \Theta}
\end{aligned}
$$

where the dimensionless lubricant film thickness, $H$, is defined as $H \equiv 1.0-R_{s / b}$. The dimensionless velocities, $U_{s}$ and $V_{s}$, at the surface of the rotating shaft are given by

$$
\begin{aligned}
U_{s}= & \frac{d E}{d \tau} \cos (\Theta-\Phi)+E \frac{d \Phi}{d \tau} \sin (\Theta-\Phi) \\
& +2 R_{s / b}^{*} \sin \left(\Theta-\Theta^{*}-2 \tau\right), \\
V_{s}= & -\frac{d E}{d \tau} \sin (\Theta-\Phi)+E \frac{d \Phi}{d \tau} \cos (\Theta-\Phi) \\
& +2 R_{s / b}^{*} \cos \left(\Theta-\Theta^{*}-2 \tau\right),
\end{aligned}
$$

where

$$
\begin{aligned}
& \Theta=\theta, \quad Z=\frac{z}{R_{b}}, \quad L=\frac{l}{R_{b}}, \quad P=\frac{p}{P_{a}}, \\
& R_{s / b}=\frac{R_{s}}{R_{b}}, \quad \sigma=\frac{\omega \eta}{p_{a}}, \\
& U_{s}=\frac{2 u_{s}}{R_{b} \omega}, \quad V_{s}=\frac{2 v_{s}}{R_{b} \omega}, \quad R_{s / b}^{*}=\frac{R_{s}^{*}}{R_{b}}, \\
& \tau=\frac{\omega t}{2}, \quad E=\frac{e}{R_{b}}, \quad \Phi=\phi .
\end{aligned}
$$

In the study, a spectral finite difference method is applied for solving the pressure distribution equation, the lubricated film thickness, $H$, is expressed in a Fourier series to the circumferential $\Theta$-direction, and (1) is decomposed into each component of the Fourier series as shown in (4a)-(4d), so that (1) can be integrated with respect to time independently of each component to get a steady-state solution. This process results in high resolution in space and high speed in computation as follows:

$$
H(Z, \Theta, \tau)=\sum_{n=0}^{\infty} H_{c n}(Z, \tau) \cos n \Theta+\sum_{n=1}^{\infty} H_{s n}(Z, \tau) \sin n \Theta,
$$

$U_{s}(Z, \Theta, \tau)=\sum_{n=0}^{\infty} U_{c n}(Z, \tau) \cos n \Theta+\sum_{n=1}^{\infty} U_{s n}(Z, \tau) \sin n \Theta$,

$$
\begin{array}{r}
V_{s}(Z, \Theta, \tau)=\sum_{n=0}^{\infty} V_{c n}(Z, \tau) \cos n \Theta+\sum_{n=1}^{\infty} V_{s n}(Z, \tau) \sin n \Theta, \\
P(Z, \Theta, \tau)=\sum_{n=0}^{\infty} P_{c n}(Z, \tau) \cos n \Theta+\sum_{n=1}^{\infty} P_{s n}(Z, \tau) \sin n \Theta .
\end{array}
$$

At $Z=0$, the groove shape is symmetric, the boundary conditions of pressure are

$$
\begin{array}{ll}
\frac{\partial P_{c n}}{\partial Z}=0 \quad & (n \geq 0), \\
\frac{\partial P_{s n}}{\partial Z}=0 \quad(n \geq 1),
\end{array}
$$

and it is assumed that the fluid is open to the atmosphere at $Z=L$, so that boundary conditions of pressure are

$$
\begin{gathered}
P_{c n}=1.0 \quad(n=0), \\
P_{c n}=0, \quad P_{s n}=0 \quad(n \geq 1) .
\end{gathered}
$$

And in the numerical procedure, in order to accurately represent the geometry of lubricant film in the Fourier series, according to the sampling theorem [8], the Fourier series terms should take a half of the total number of geometry data in the circumferential direction as shown in Figure 3. 


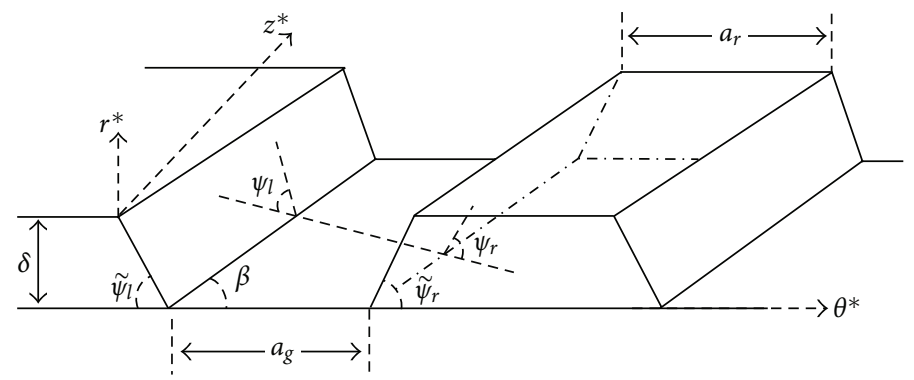

Figure 2: The trapezoidal groove.
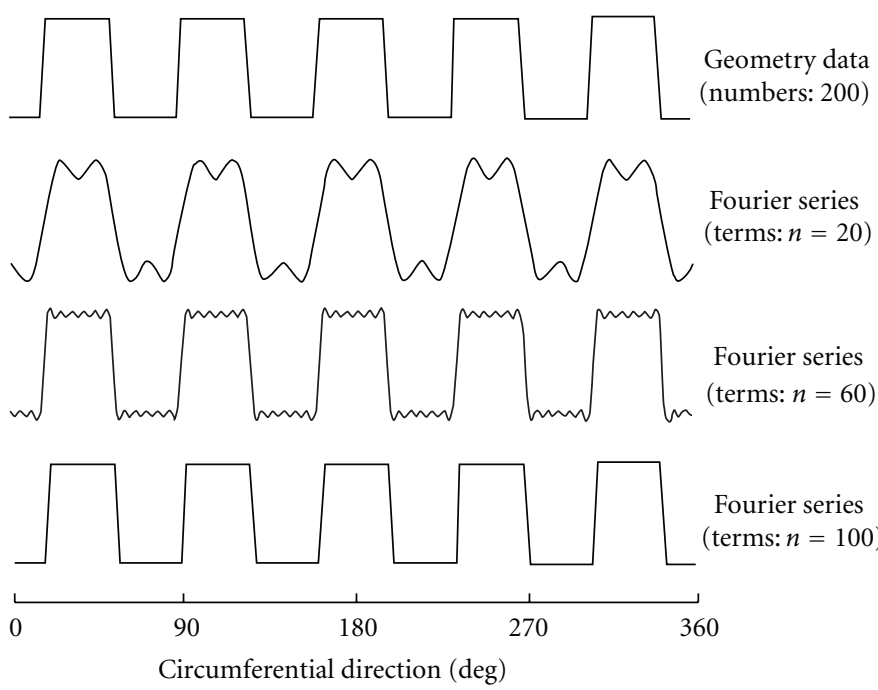

FIGURE 3: Lubricant film geometry in the Fourier series.

\section{Load Capacity, Attitude Angle, and Friction Torque}

The dimensionless radial component of load force, $F_{r}$, tangential component of load $F_{t}$, and friction torque of shaft, $T_{f}$, are

$$
\begin{aligned}
F_{r}= & 2 \int_{0}^{L} \int_{0}^{2 \pi}\left[f_{R^{*}}^{d s} \frac{\cos \left(\Theta^{*}+\Phi\right)}{\cos \psi} R_{s / b}^{*}\right] d \Theta^{*} d Z^{*} \\
& -2 \int_{0}^{L} \int_{0}^{2 \pi}\left[f_{\Theta^{*}}^{d s} \frac{\sin \left(\Theta^{*}+\Phi\right)}{\cos \psi} R_{s / b}^{*}\right] d \Theta^{*} d Z^{*}, \\
F_{t}= & 2 \int_{0}^{L} \int_{0}^{2 \pi}\left[f_{R^{*}}^{d s} \frac{\sin \left(\Theta^{*}+\Phi\right)}{\cos \psi} R_{s / b}^{*}\right] d \Theta^{*} d Z^{*} \\
& +2 \int_{0}^{L} \int_{0}^{2 \pi}\left[f_{\Theta^{*}}^{d s} \frac{\cos \left(\Theta^{*}+\Phi\right)}{\cos \psi} R_{s / b}^{*}\right] d \Theta^{*} d Z^{*}, \\
& T_{f}=-2 \int_{0}^{L} \int_{0}^{2 \pi}\left[\frac{f_{\Theta^{*}}^{d s}}{\cos \psi} R_{s / b}^{* 2}\right] d \Theta^{*} d Z^{*},
\end{aligned}
$$

where $f_{R^{*}}^{d s}$ and $f_{\Theta^{*}}^{d s}$ are the $R^{*}$ - and $\Theta^{*}$-components of fluid force per unit area on the surface of shaft, which can be obtained as referenced in Liu and Mochimaru [9].
Then the dimensionless load capacity, $W$, is given by

$$
W=\sqrt{F_{r}^{2}+F_{t}^{2}}
$$

and the attitude angle, $\varphi$, is

$$
\varphi=\tan ^{-1}\left(\frac{F_{t}}{F_{r}}\right) .
$$

\section{Results and Discussion}

In case of $n_{g}=5, \beta=30 \mathrm{deg}, a_{r} / a_{g}=1.0, \delta=C_{r}, C_{r} / R_{b}=$ $0.005, \Phi=0, L=1.0, \Omega=0$, and $\Lambda \equiv \sigma \times\left(R_{s} / C_{r}\right)^{2}=1.0$, numerical results are shown in Figures 4-8.

Figure 4 displays an isobar diagram in the $\Theta^{*} Z^{*}$-plane with eccentricity changes, it shows that the pressure gradient in $\Theta^{*}$ - and $Z^{*}$-direction is not zero even in the case of eccentricity equal to zero, which confirm the fact that the herringbone grooves have the pumping effect. And Figure 5 gives the pressure distribution with trapezoidal angle increases, as long as the trapezoidal angle is smaller than $30 \mathrm{deg}$, the high pressure zone is slightly increased with trapezoidal angle increase, but in cases that the groove angle is larger than $30 \mathrm{deg}$, the high pressure zone decreases and the lower pressure zone increases with an increase of trapezoidal angle. 


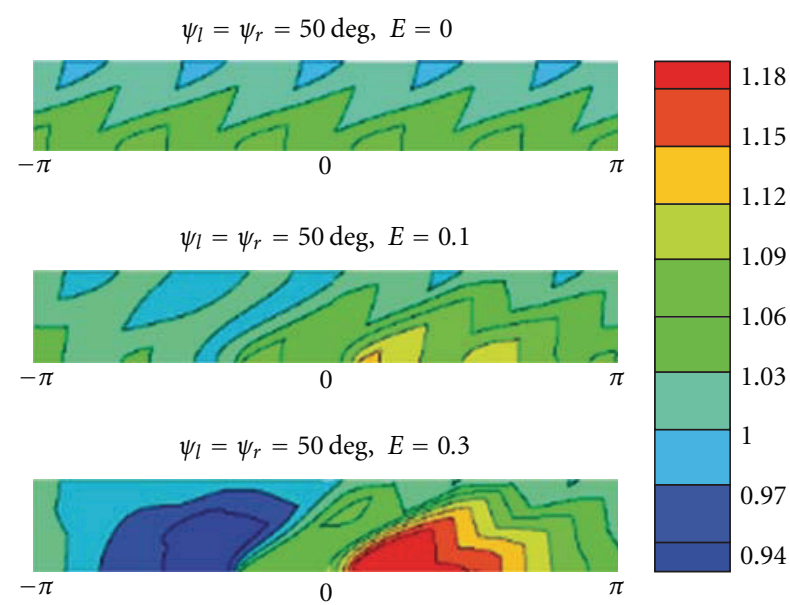

FIGURE 4: Isobar diagram with eccentricity.

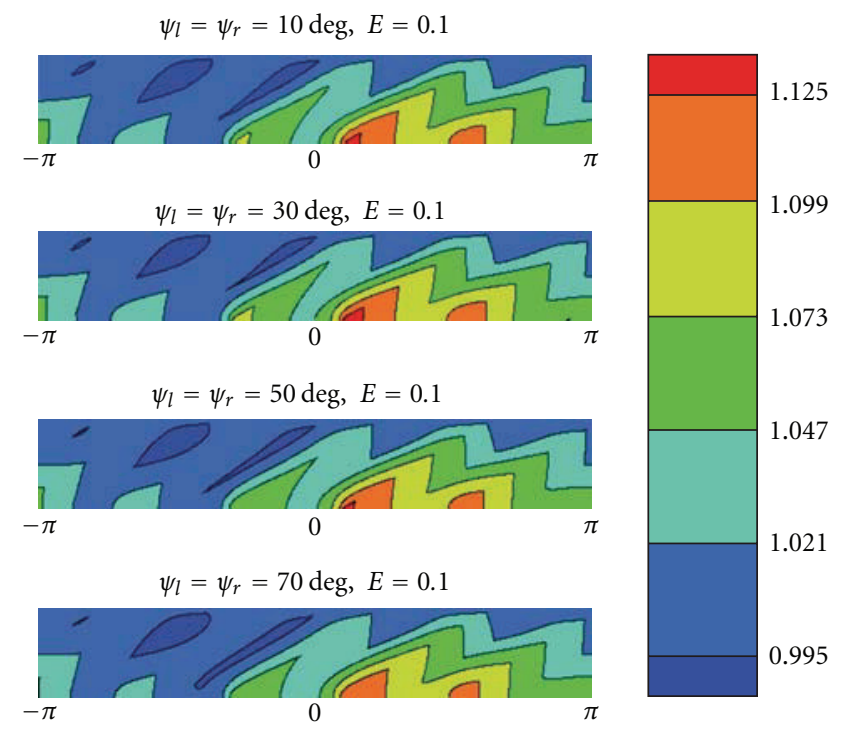

FIGURE 5: Isobar diagram with trapezoidal angle.

There are three types of asymmetric trapezoidal grooves as shown in Figure 6. Figure 7(a) presents an isobar diagram in the $\Theta^{*} Z^{*}$-plane, and the circumferential pressure distribution at the center of the shaft (at $Z^{*}=0$ ) is shown in Figure 7(b), where the dashed line is for the pressure distribution of the plane journal bearing case. In addition, the load capacity, attitude angle, and friction torque with trapezoidal angle changes are evaluated as shown in Figure 8, it shows that the lower pressure zone is the least area in the case of type 3, which is a favorable status to prevent cavitation, but in the case of type 1 , the radial load capacity is maximum and the friction torque is minimum; it is useful for application on the miniaturization electronic device.

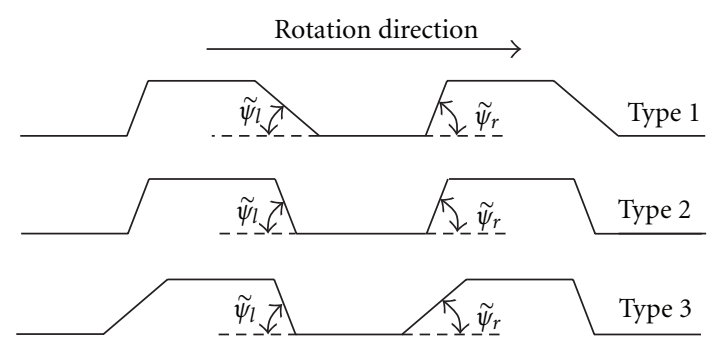

FIGURE 6: Asymmetric trapezoidal groove.

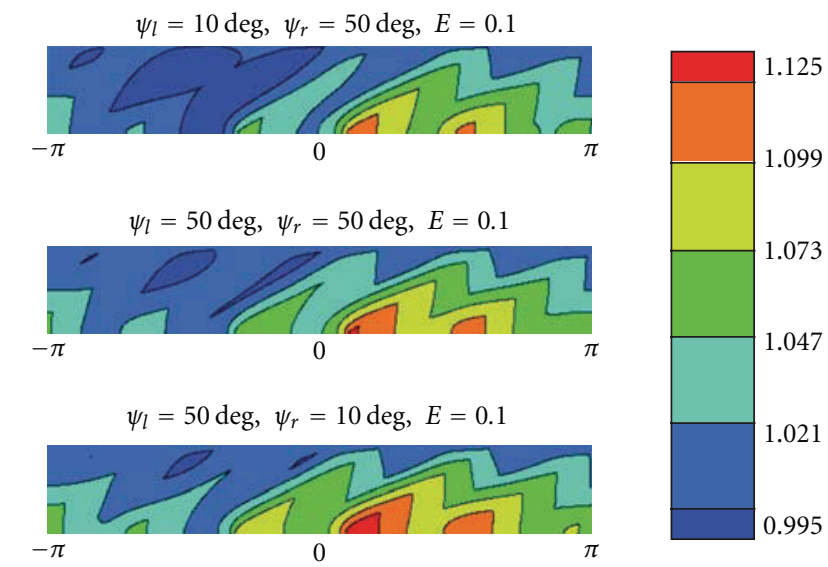

(a)

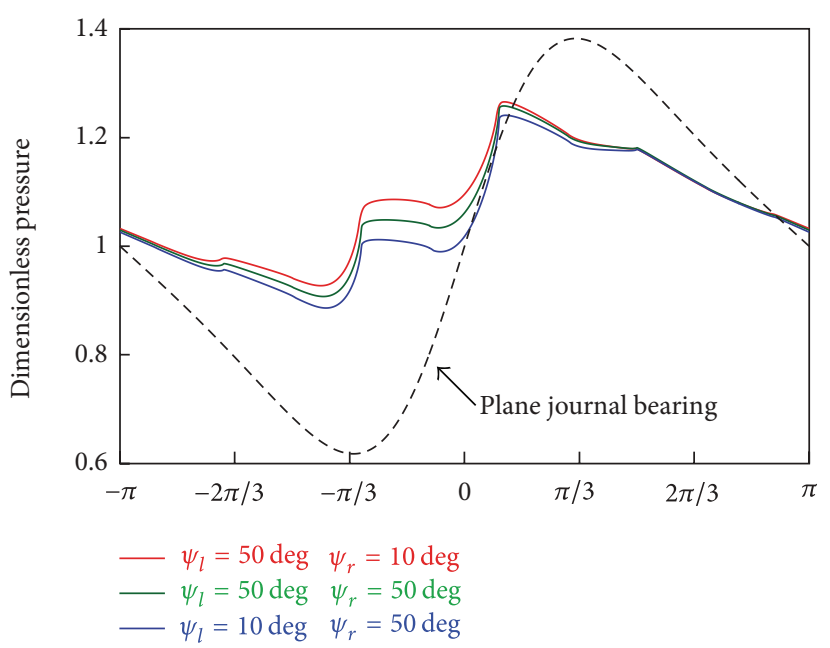

(b)

Figure 7: (a) Isobar diagram of pressure distribution. (b) Circumferential pressures distribution at $Z^{*}=0$.

\section{The Optimal Parameters of Trapezoidal Groove Geometry}

Comparing with plane journal bearing, groove journal bearing has some distinguished advantages such as lower noise, higher stability but also has disadvantages such as small radial load capacity and larger friction torque. The optimal 


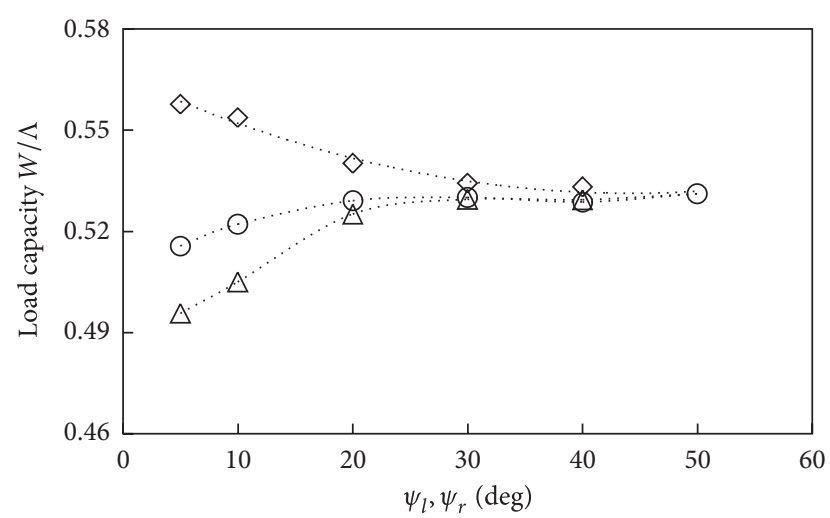

$\diamond \psi_{l}=50 \mathrm{deg}, \psi_{r}=5,10,20,30,40,50 \mathrm{deg}$

$\bigcirc \psi_{l}=\psi_{r}=5,10,20,30,40,50 \mathrm{deg}$

$\Delta \psi_{l}=5,10,20,30,40,50 \mathrm{deg}, \psi_{r}=50 \mathrm{deg}$

(a)

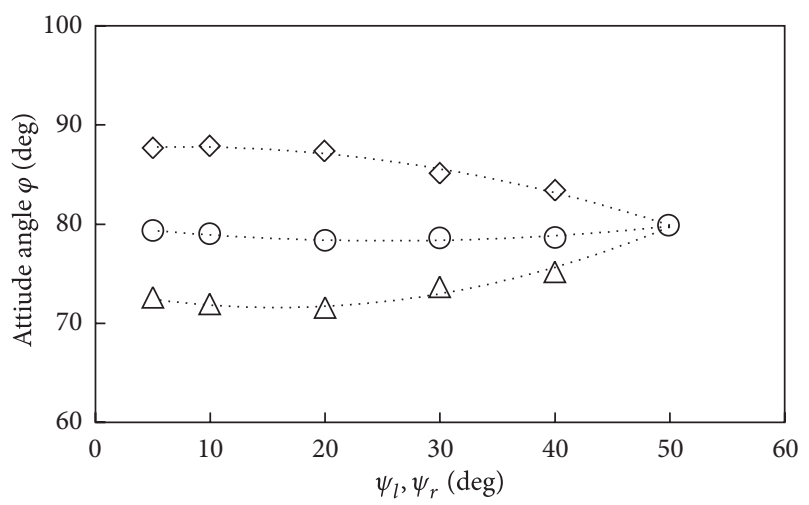

$\diamond \psi_{l}=50 \mathrm{deg}, \psi_{r}=5,10,20,30,40,50 \mathrm{deg}$

$\psi_{l}=\psi_{r}=5,10,20,30,40,50 \mathrm{deg}$

$\triangle \psi_{l}=5,10,20,30,40,50 \mathrm{deg}, \psi_{r}=50 \mathrm{deg}$

(b)

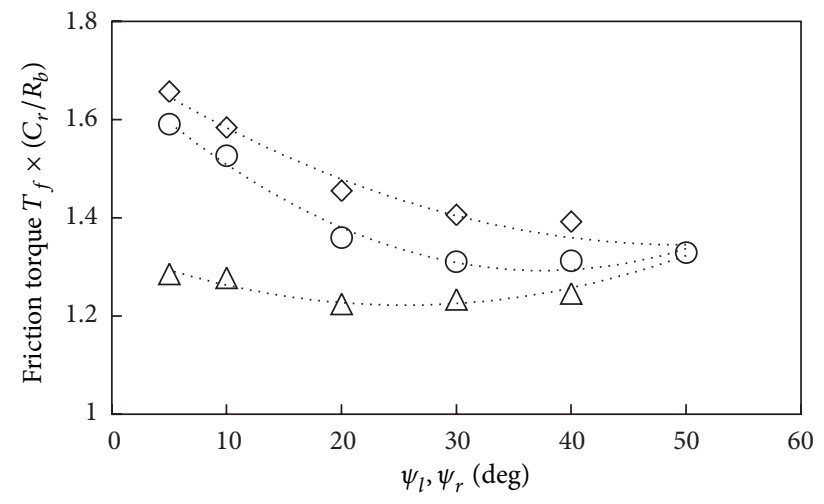

$\diamond \psi_{l}=50 \mathrm{deg}, \psi_{r}=5,10,20,30,40,50 \mathrm{deg}$

$\psi_{l}=\psi_{r}=5,10,20,30,40,50 \mathrm{deg}$

$\triangle \psi_{l}=5,10,20,30,40,50 \mathrm{deg}, \psi_{r}=50 \mathrm{deg}$

(c)

FIGURE 8: (a) Load capacities with trapezoidal angle. (b) Attitude angles with trapezoidal angle. (c) Friction torque with trapezoidal angle.

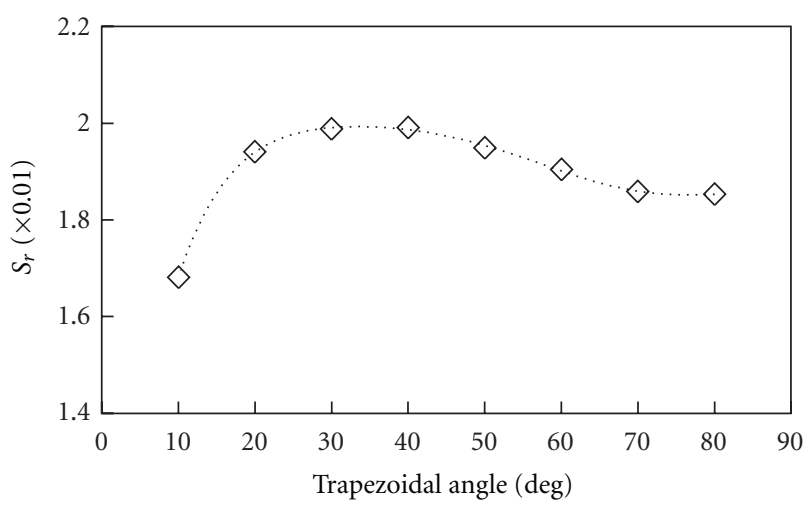

FIGURE 9: Estimation $S_{r}$ with trapezoidal angle.

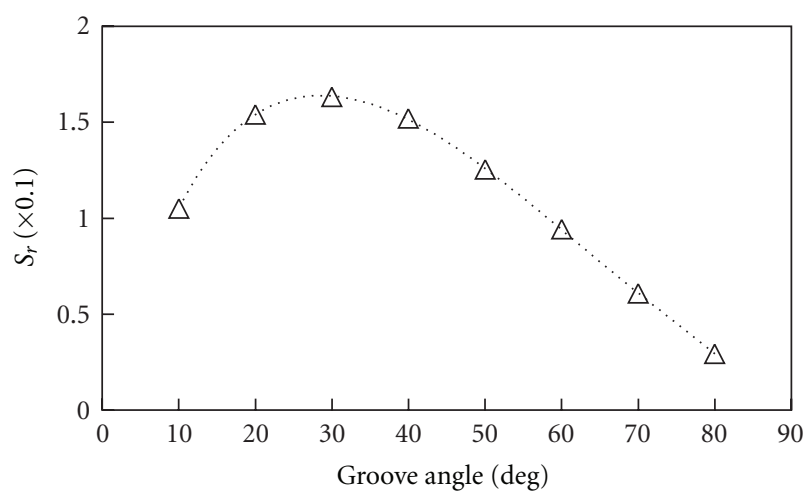

FIGURE 10: Estimation $S_{r}$ with groove angle.

trapezoidal groove geometry can be estimated by the value, $S_{r}$, which is given by the ratio of radial load capacity to friction torque as

$$
S_{r} \equiv \frac{F_{r} / \Lambda}{T_{f} \times\left(C_{r} / R_{b}\right)} .
$$

The common parameters of groove geometry are given as $n_{g}=5, a_{r} / a_{g}=1.0, e=0.1 C_{r}, C_{r} / R_{b}=0.005, \Phi=0, L=$ 1.0, $\Omega=0$, and $\Lambda=1.0$. Figure 9 presents $S_{r}$ with respect to trapezoidal angle; in case of $\beta=30 \mathrm{deg}, \delta=C_{r}$, and $\psi_{l}=\psi_{r}$, it shows the optimal trapezoidal angle is $20 \mathrm{deg} \sim 50 \mathrm{deg}$.

In case of $\delta=C_{r}$, and $\psi_{1}=\psi_{r}=30 \mathrm{deg}, S_{r}$ with the groove angle of values from 10 to 80 degrees are evaluated as a function of $\beta$ in Figure 10, which shows that when grooves angle $\beta$ is approximately 30 degree; $S_{r}$ has a maximum value.

Moreover, in case of $\beta=30 \mathrm{deg}, \psi_{l}=\psi_{r}=30 \mathrm{deg}$, the most suitable groove depth, $\delta$, is 1.4 times of the bearing clearance $C_{r}$ for the maximum value $S_{r}$ as shown in Figure 11 .

\section{Conclusions}

The pressure distributions generated along a fluid-lubricated herringbone-grooved journal bearing with trapezoidal groove are investigated at several cases of trapezoidal angles of groove. Regarding $S_{r}$, which is given by the ratio of radial 


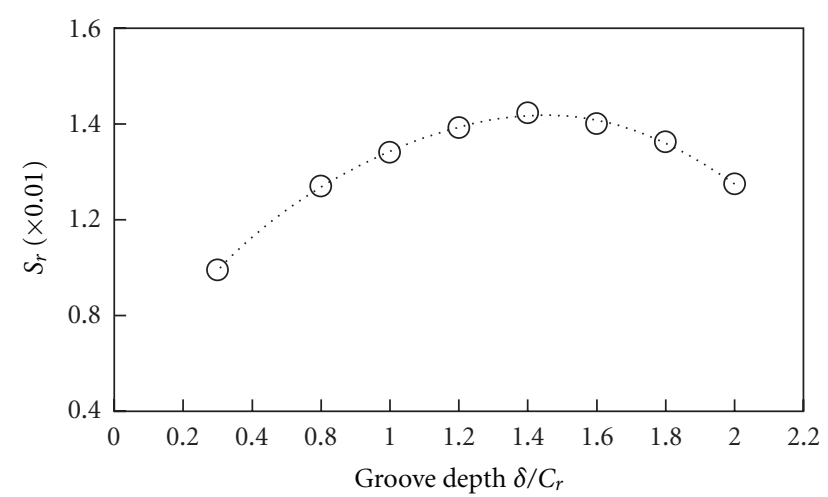

FIGURE 11: Estimation $S_{r}$ with groove depth.

load capacity to friction torque, in the case of $n_{g}=5$, $a_{r} / a_{g}=1.0, e=0.1 C_{r}, C_{r} / R_{b}=0.005, \Phi=0, \stackrel{L}{L}=1.0$, $\Omega=0$, and $\Lambda=1.0$, the optimal parameters of trapezoidal grooves geometry are found as follows.

(1) The trapezoidal angle is $20 \mathrm{deg} \sim 50 \mathrm{deg}$.

(2) The groove depth is 1.4 times of the bearing clearance.

(3) The grooves angle is approximately 30 degree.

\section{Nomenclature}

$a_{g}, a_{r}:$ Grooves width, ridge width

$C_{r}$ : Bearing clearance

$e, E:$ Eccentricity, dimensionless eccentricity

$f_{R^{*}}^{d s}$ : Dimensionless $R^{*}$-component of fluid force per unit area which acts on surface of shaft

$f_{\Theta^{*}}^{d s}:$ Dimensionless $\Theta^{*}$-component of fluid force per unit area which acts on surface of shaft

$F_{r}: \quad$ Dimensionless radial component of load

$F_{t}$ : Dimensionless tangential component of load

$H: \quad$ Dimensionless fluid film thickness

$l, L$ : $\quad$ Bearing length, dimensionless bearing length

$n_{g}: \quad$ Number of grooves

$P$ : $\quad$ Dimensionless pressure

$P_{a}: \quad$ Atmospheric pressure

$r, \theta, z$ : Inertial coordinates

$R_{b}$ : Radius of bearing

$R_{s 0}$ : Radius of shaft without grooves

$R_{s}$ : Radial component of coordinate at surface of shaft

$R_{s / b}$ : Dimensionless radial component of coordinate at surface of shaft

$S_{r}: \quad$ Estimation value

$t, \tau$ : Time, dimensionless time

$\begin{array}{ll}T_{f}: & \begin{array}{l}\text { Dimensionless friction torque of rotating } \\ \text { shaft }\end{array} \\ U_{s}: & \begin{array}{l}\text { Dimensionless circumferential velocity at } \\ \text { surface of rotating shaft }\end{array} \\ V_{s}: & \begin{array}{l}\text { Dimensionless radial velocity at surface of } \\ \text { rotating shaft }\end{array} \\ W: & \text { Dimensionless load capacity of bearing } \\ \beta: & \text { Groove angle } \\ \delta: & \text { Groove depth, dimensionless groove depth } \\ \eta: & \text { Viscosity of fluid } \\ \Lambda: & \text { Bearing number } \\ \sigma: & \text { Dimensionless number } \\ \varphi: & \text { Attitude angle of shaft } \\ \psi: & \text { Trapezoidal angle of groove } \\ \phi: & \text { Angle between the fixed axis of abscissa } \\ & \text { ( } \theta=0) \text { and the axis of eccentricity } \\ \Phi: & \text { Dimensionless angle between the fixed } \\ & \text { axis of abscissa }(\theta=0) \text { and the axis of } \\ & \text { eccentricity } \\ \omega: & \text { Rotation velocity of shaft } \\ \Omega: & \text { Swirl velocity of shaft } \\ \text { Superscript } & *:\end{array}$

\section{References}

[1] J. H. Vohr and C. H. T. Pan, "On the spiral-grooved, self-acting gas bearing," Tech. Rep. MTI63TR52, 1963.

[2] H. S. Cheng and C. H. T. Pan, "Stability analysis of gaslubricated, self-acting, plain, cylindrical, journal bearings of finite length, using Galerkin's method,' Journal of Basic Engineering, vol. 87, no. 1, pp. 185-192, 1965.

[3] B. J. Hamrock and D. P. Fleming, "Optimization of self-acting herringbone grooved journal bearings for maximum radial load capacity," NASA-TM-X-52945, 1971.

[4] S. Murata, Y. Miyake, and N. Kawabata, "Two-dimensional analysis of herringbone groove journal bearing," Bulletin of the JSME, vol. 23, no. 181, pp. 1980-1987, 1980.

[5] D. Bonneau and J. Absi, "Analysis of aerodynamic journal bearings with small number of herringbone grooves by finite element method," Journal of Tribology, vol. 116, no. 4, pp. 698-704, 1994.

[6] C. C. Rondonuwu and S. H. Winoto, "Pressure distributions along vertical hydrodynamic herringbone-grooved journal bearings," Tribology Transactions, vol. 49, no. 2, pp. 174-181, 2006.

[7] J. Liu and Y. Mochimaru, "Analysis of oil-lubricated herringbone grooved journal bearing with trapezoidal cross-section, using a spectral finite difference method," Journal of Hydrodynamics $B$, vol. 22, supplement 1, no. 5, pp. 408-412, 2010.

[8] H. Nyquist, "Certain topics in telegraph transmission theory," AIEE Transaction, vol. 47, pp. 617-644, 1928.

[9] J. Liu and Y. Mochimaru, "The effects of trapezoidal groove on a self-acting fluid-lubricated herringbone grooves journal bearing," ISRN Tribology, vol. 2013, Article ID 240239, 7 pages, 2013. 

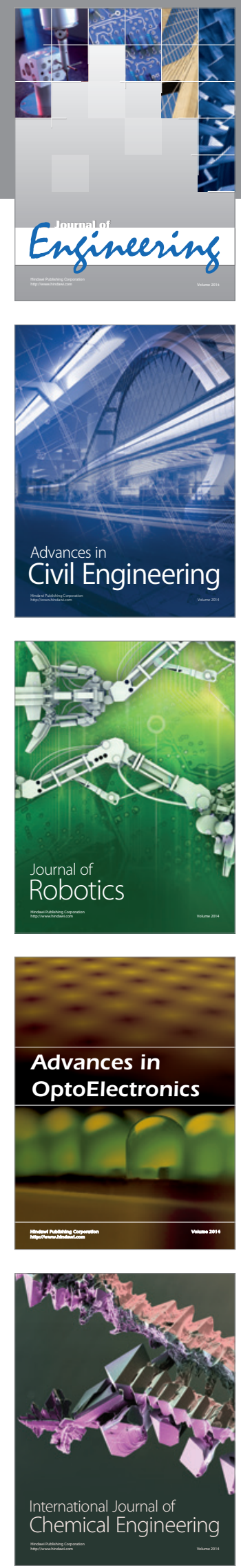

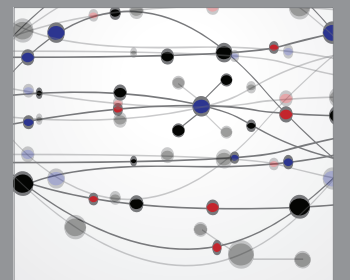

The Scientific World Journal
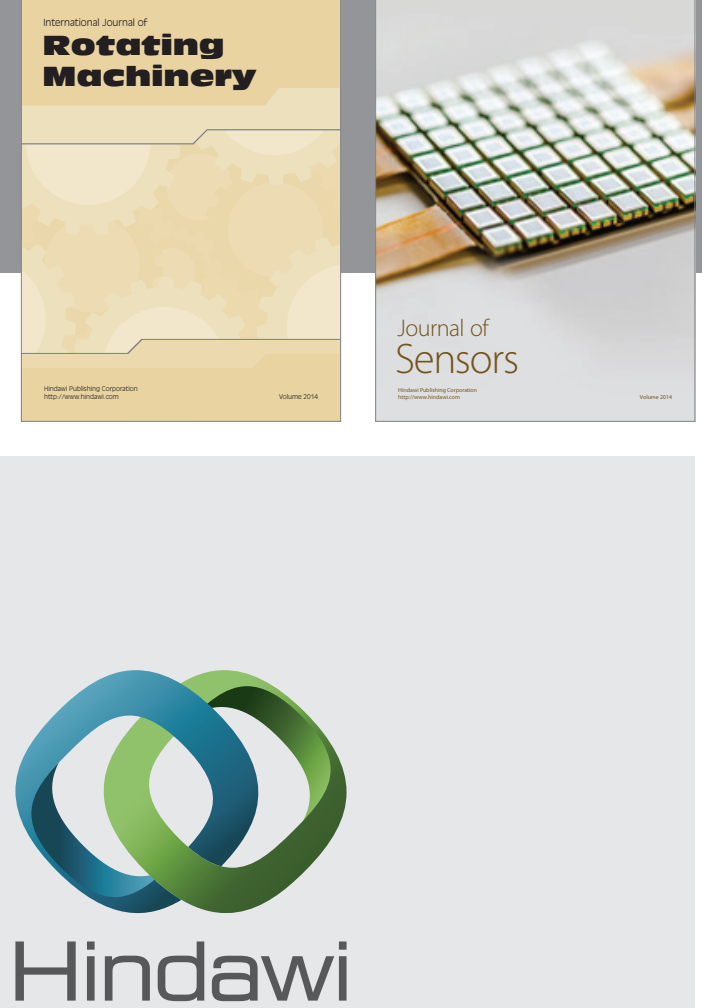

Submit your manuscripts at http://www.hindawi.com
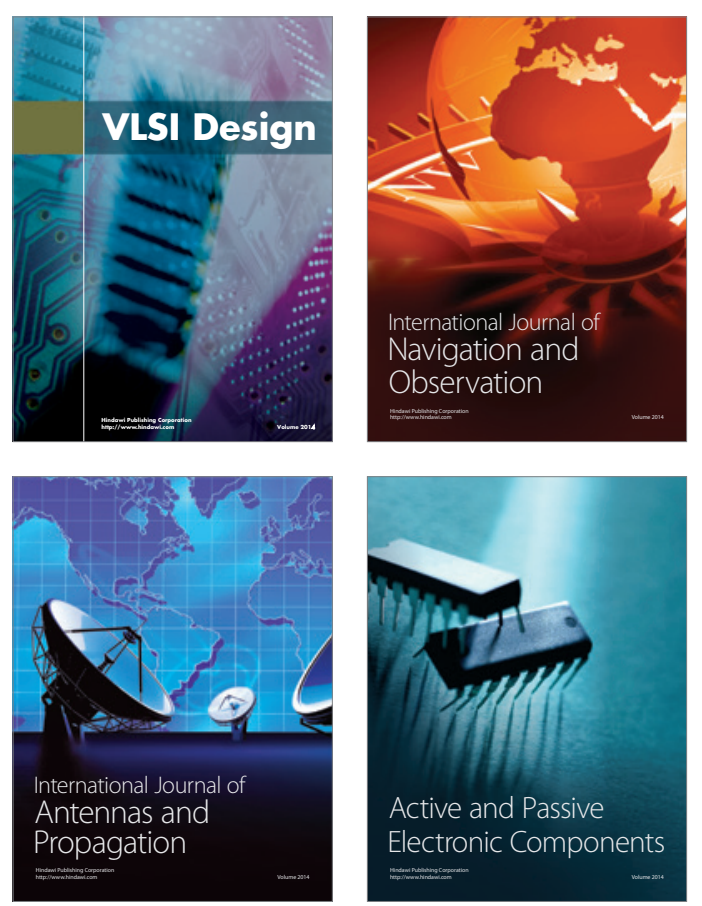
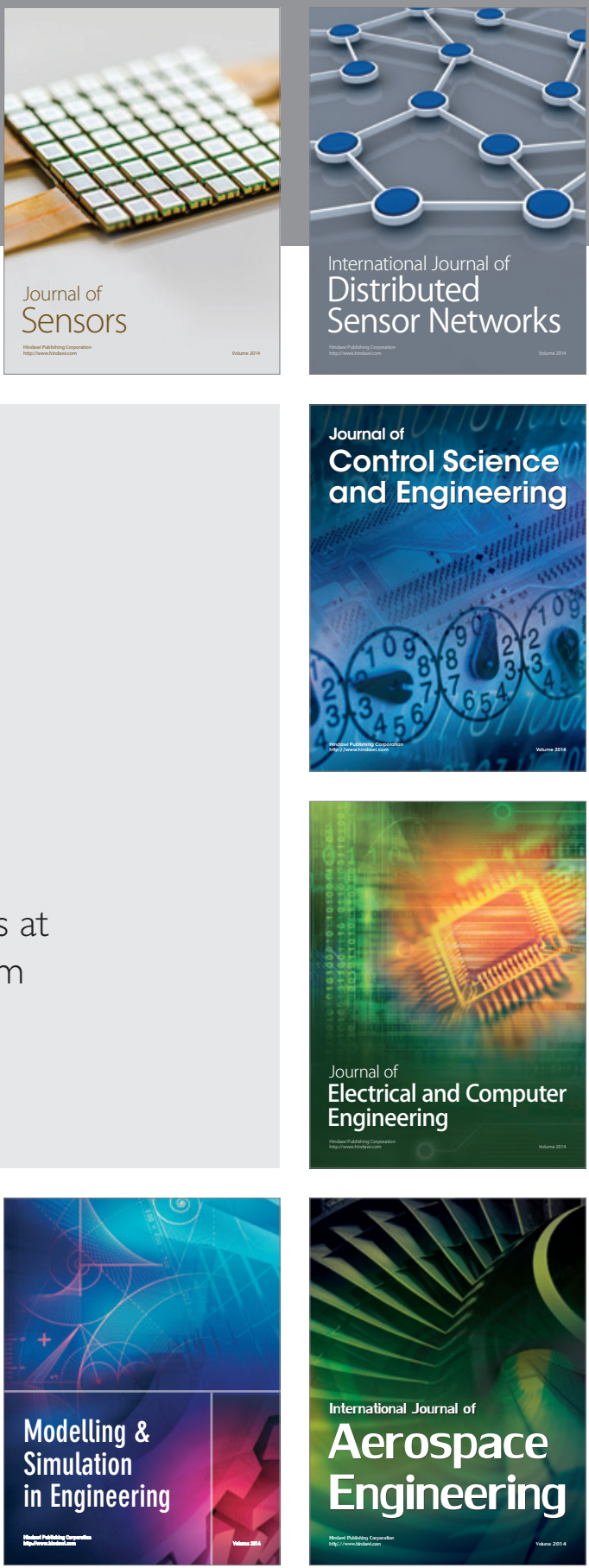

Journal of

Control Science

and Engineering
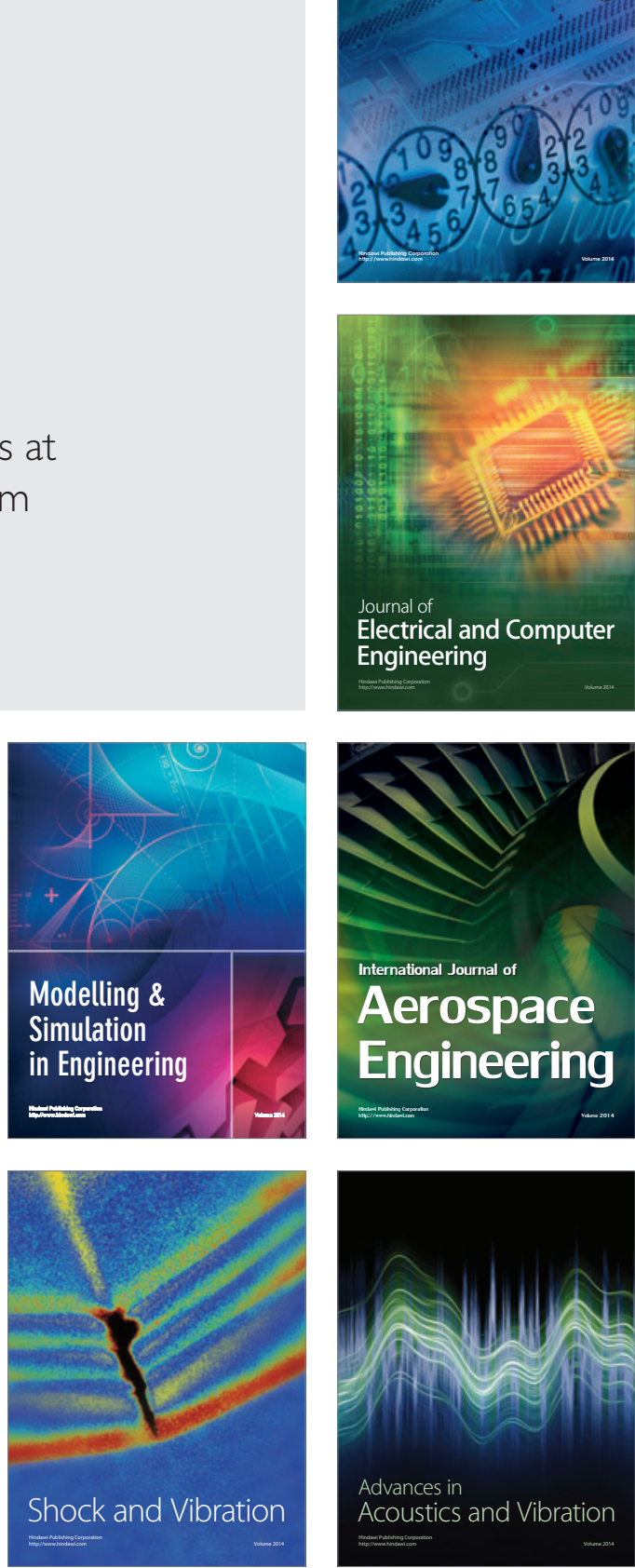